\title{
DDT-modulated behavioural profile in terms of longevity, fecundity fertility and fitness in subsequent generations of DDT-resistant Phlebotomus argentipes (Diptera: Psychodidae)
}

\author{
Aarti Rama ${ }^{1}$, Pradeep Das ${ }^{2}$ and Vijay Kumar ${ }^{1, *}$ \\ ${ }^{1}$ Department of Vector Biology and Control and \\ ${ }^{2}$ Department of Molecular Biology, ICMR-Rajendra Memorial Research Institute of Medical Sciences, Agamkuan, Patna 800007 , India
}

Dichlorodiphenyltrichloroethane (DDT), a chlorinated insecticide served as a well-established tool against the Indian Visceral leishmaniasis (VL) or kala-azar vector, i.e. Phlebotomus argentipes (Diptera: Psychodidae) till infestation with insecticide resistance (IR) among these vector species, leading to the legacy of failure of major campaigns for global VL elimination via vector control strategy. Failing in the task of controlling the insect population, IR has definitely imposed a negative impact over the quality of mosquito vector species in terms of their physiological fitness and reproductive viability, but this has never been reported in the case of Indian $V L$ vector. Therefore, to explore IR implications and comparative assessment over the physiological fitness and reproductive robustness within subsequent generations of laboratory-reared resistant sand flies, bioassay experiments have been performed up till the seventh generation of homozygous 4\% DDT-resistant strained colony (DRC) of $P$. argentipes. With observed mean vector longevity ranging between 3 and 9 days (for male sand flies) as well as 5 and 13.5 days (for female sand flies) till their seventh generation, IR causes an abrupt decrease in fecundity (with mean potential fecundity per female from 60.72 to 6.44), fertility (with mean no. of eggs oviposited per female from 53.4 to 6.02 and mean no. of eggs hatching per female from 13.6 to 1.67) and greatly affecting reproductive fitness and behaviour throughout the studied generations of DRC sand flies. The affected DDT-modulated behavioural profile in terms of the studied four-pronged LF3 parameters (i.e. longevity, fitness, fecundity and fertility), revealed that the changed quality of DRC $P$. argentipes in the forthcoming generations to such an extent, that it might prove to be detrimental to the survival and fitness of the sand flies, if selection pressures maintained throughout their subsequent generations. The results of the present study provide the probable reasons and side effects associated with the developed resistance both at macroscopic and microscopic levels, which

*For correspondence. (e-mail: vijayrnagar@hotmail.com) deserve additional attention for uprooting the disease from its end.

Keywords: Behavioural responses, Dichlorodiphenyltrichloroethane, insecticide resistance, Phlebotomus argentipes, Visceral leishmaniasis.

INDIAN female sand flies Phlebotomous argentipes (Diptera: Psychodidae) infected with protozoan parasite Leishmania donovani (Kinetoplastida: Trypanosomatidae) are the 'key transmitters' for Visceral leishmaniasis (VL) or kala-azar, being proliferated within the human population ${ }^{1}$. VL is a debilitating and often fatal disease targeting the marginalized and poor people residing in Bihar, Jharkhand, West Bengal and Uttar Pradesh ${ }^{2,3}$, among which Bihar is highly prone to VL. The current Indian VL control programmes rely on the prophylactic and therapeutic use of anti-leishmanial drugs, but vectorcontrol options are a more reliable approach.

In India, for checking the outgrowth of indoor-dwelling sand flies, Indoor Residual Spraying (IRS) with Dichlorodiphenyltrichloroethane (DDT, chlorinated insecticide) has been validated as a cost-effective vector control strategy since 1953 (refs 4-7). After the decades of extensive application of DDT-IRS, $P$. argentipes had reportedly attained tolerance/resistance against it $^{8-14}$. Since 2016, blanket covering of IRS with 5\% alphacypermethrin (synthetic pyrethroid insecticide) in the districts of Bihar, has successfully replaced DDT from the national VL vector control programme ${ }^{15}$ and fuelled the target of VL elimination by 2017 (ref. 16) now revised for 2020, and is also providing more satisfactory results in this aspect. However, logistic limitations and lower durability associated with alphacypermethrin (in the context to its efficacy as compared with DDT) ${ }^{17}$, might result in Insecticide Resistance (IR) against sand flies, reversing the insecticide-based VL vector control strategies.

Therefore, for avoiding such instances, it is essential to widen our understanding regarding the probable reasons and side effects associated with the developed resistance 
among this VL vector species at micro and macro levels. More importantly, DDT resistance affecting physiological fitness and reproductive robustness within the subsequent generations, especially in the context of Indian VL vector has not been studied till date.

The sub-lethal effects of DDT exposure upon the behavioural profile of $P$. argentipes focusing on the fourpronged LF3 parameters, viz. longevity, fecundity, fertility and fitness, have been studied in this article. The enhanced understanding of IR regulation mechanism, its cause and effect on the host would further allow detection and tracking of IR in its initial stage, and hence will further contribute in developing instant strategic rationales for long-term insecticide use for targeting vectors.

\section{Materials and methods}

For assessing IR implicating physiological fitness and reproductive robustness in the subsequent generations of $P$. argentipes, healthy and active sand flies obtained from the established 4\% DDT-resistant colony (DRC) served as the 'backbone' for this study ${ }^{12,13}$. Simultaneously, sterilized sand flies of non-resistant colony (NRC) that had never been exposed to any insecticide and were perfectly maintained for $>30$ years, served as control samples for bioassay experiments ${ }^{18}$.

Both type of sand flies, viz. DRC and NRC were housed under controlled environment, i.e. $28^{\circ} \pm 2{ }^{\circ} \mathrm{C}$; $80 \% \pm 5 \% \mathrm{RH}$ and $12: 12(\mathrm{~L}: \mathrm{D})$ hours photoperiod in the insectarium at the Department of Vector Biology and Control, ICMR-Rajendra Memorial Research Institute of Medical Sciences (RMRIMS), Patna, Bihar ${ }^{12,13}$. Under the sandfly colony maintenance procedure, healthy and mature male rabbits Oryctolagus cuniculus (Legomorpha: Leporidae) were used for providing blood meal to the freshly emerged female sand flies ${ }^{19,20}$.

The rabbits were reared at the animal house of ICMRRMRIMS, by strictly following 'The Principles of laboratory animal care (NIH publication no. 85-23, revised 1985) as well as the protocols of the Animal Ethics Committee, ICMR, Government of India (GoI) for conducting experiments.

\section{Behavioural transition in terms of LF3 propounded by $D D T$}

This has been validated with sets of bioassay experiments by focusing on the four-pronged LF3 parameters for DRC sand flies. Among these parameters, longevity, fecundity and fertility have been assessed and compared within the subsequent generations (till seventh generation) during 2013-2015 along with the process of procuring and establishment of homozygous DRC of $P$. argentipes ${ }^{12}$. Whereas bioassay experiment for assessing fitness of resistant sand flies was validated with flies from randomly selected generations.
Experiment 1: Susceptibility confirmation and longevity analysis of resistant sand flies: Prior to conducting bioassay experiments with F1 generation, susceptibility of parents as well as progenies of DRC of $P$. argentipes was ascertained by following the protocol of insecticide susceptibility test against 4\% DDT-impregnated papers ${ }^{21}$. These insecticide-impregnated papers (prepared in coordination with the World Health Organization by the Vector Control Research Unit, Universiti Sains Malaysia, Penang, Malaysia in 2013) were procured from the National Vector Borne Disease Control Programme (NVBDCP), GoI, in 2013 for conducting the bioassay. The susceptibility of colonized sand flies at each subsequent generation was observed in terms of mortality frequency rate (MFR) and DDT resistant rate (DRR), as discussed earlier ${ }^{12}$.

After susceptibility confirmation, tested female sand flies were offered blood meals from the healthy, caged, male rabbit hosts and were allowed to feed over them until satiated $^{12}$. After completion of blood-feeding process and post-feeding acclimatization, the engorged female along with male sand flies were carefully transferred to the oviposition-cum-rearing pots (Hilton pots), designed for laying eggs and maintenance of its generation. These pots were facilitated with sprung pieces of filter paper for retaining moisture from the plastered surface and on which eggs were laid by the female insects following copulation with male insects. The oviposition pots were placed over a damp and moist cotton cloth for constant regulation of moisture for the ovipositing female sand flies as well as for the expected brood, and were allowed to rest at the earlier mentioned controlled environment of the insectarium. These oviposition pots were examined daily for the death of confined parent insects and eggs laid by them. As soon as the hatchings were observed, usually after about 5-6 days of confinement (date marked on the oviposition pots), a small amount of finely ground, anti-fungal-treated larval food was sprinkled onto the immature stages that were further reared to adults following general procedures ${ }^{20,22,23}$.

The longevity of adult DRC sand flies for each generation was assessed by recording the mean number of days from adult emergence (in their F1 as well as their subsequent generations) till death. Observations for longevity of DRC sand flies followed by insecticide exposure and comparing them with the sterile sand flies, i.e. NRC for both the sexes would help in further accomplish analysis of IR-implicated responses with respect to fecundity, fertility and fitness.

Experiment 2: Fecundity and fertility in subsequent generations of resistant sand flies: After susceptibility confirmation and longevity assessment, DDT-exposed sand flies were further probed for analysing fecundity and fertility in subsequent generations (till the seventh generation). 


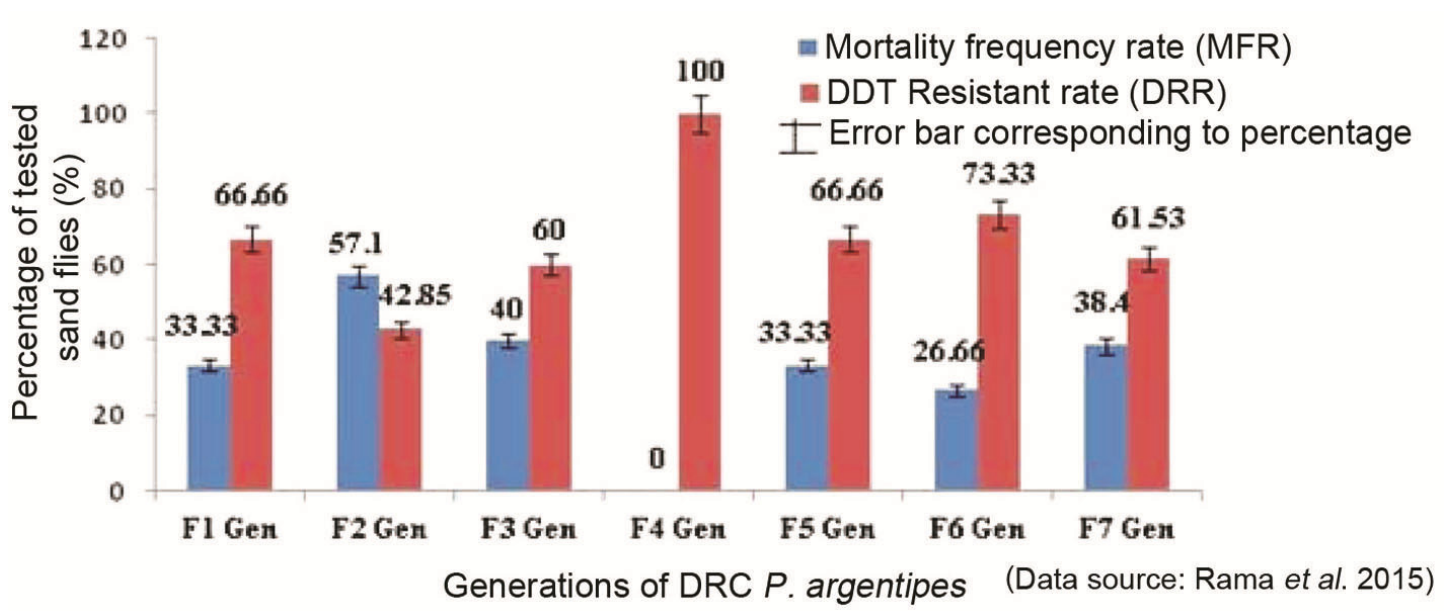

Figure 1. Comparison-based susceptibility status in terms of DDT resistant rate (DRR) and mortality frequency rate (MFR) in subsequent generation of DDT-resistant colony (DRC) Phlebotomus argentipes (adapted from Rama et al.).

Dissection of dead female insects along with microscopic observation of eggs oviposited by them was carried out under a stereoscopic microscope (Carl Zeiss Stereoscopy Microscope, Austria; model 426126), following the technique for ascertaining their parity sta$\operatorname{tus}^{24,25}$. Whereas, the total number of eggs laid and successfully hatched out as first stage larvae (L1) per experimented female sandfly studied satisfied the fertility of DRC sand flies.

Data regarding the assessment of fecundity and fertility of DRC as well as NRC sand flies along with their features, viz. egg follicles developed per female, eggs oviposited, eggs retained, hatched eggs and those remaining unhatched throughout the experiment were recorded and compared between the subsequent generations till the seventh generation. Descriptive statistical analyses were also performed using the Excel Worksheet of Microsoft ${ }^{\circledR}$.

Experiment 3: Fitness assessment of DDT-resistant strained sand flies: Fitness profile of DDT-resistant sand flies was assessed by monitoring the survivorship and growth trends of pre-adult stages in the form of their life table, including details of larvae, pupae, adult emergence at each subsequent generation following suitable methodologies ${ }^{20,26,27}$.

Experiment 4: Feeding pattern and post-feeding survivorship of resistant sand flies: In a separate experiment, 50 sand flies comprising male and female in equal proportion, enduring DDT exposure via 4\% DDT impregnated papers successfully, were released into two different cages; the first one being facilitated with healthy male rabbit as a blood meal source and the other with $10 \%$ sucrose solution-soaked cotton pad as sucrose meal. Both batches of sand flies, post-insecticide exposure, were allowed to feed on their preferred nourishment under similar laboratory environment ${ }^{18,28}$. The feeding pattern of resistant sand flies was observed at different timescales, viz. third, sixth, 12th, 24th and 36th hour of completion of susceptibility test of sand flies against DDT.

After meal supplementation, the visually recognizable sucrose-fed male, sucrose-fed female and blood-fed female DRC and NRC sand flies were confined separately into transparent, plaster-lined oviposition pots for evaluating their survivorship under controlled condition ${ }^{12-20}$. The mean results of 300 sandfly feeding patterns, confirmed with three experimental replicates, were pooled together for statistical analysis using Excel and for significance analysis using $0.05 \%$ Duncan's multiple range test (DMRT).

\section{Results}

\section{Susceptibility confirmation and longevity analysis}

Under susceptibility assessment, $P$. argentipes in the subsequent generations (till the seventh generation) were placed under selection pressure and the resistant strain was tested for susceptibility towards DDT. The DRC sand flies exhibited $33.33 \%, 57.10 \%, 40 \%, 0 \%, 33.33 \%$, $26.66 \%$ and $38.41 \%$ MFR and as $66.66 \%, 42.85 \%, 60 \%$, $100 \%, 66.66 \%, 73.33 \%$ and $61.53 \%$ DRR from first generation to the seventh generation, following their exposure to DDT. Significant fluctuation within DRR $(67.29 \% \pm 17.28 \% ; \quad$ CI $=54.49-80.07) \quad$ was observed throughout the seventh generation. MFR being inversely proportional to DRR, denoted the susceptibility of the insects and hence zero susceptibility indicated absolute resistance in the fourth generation. Maximum susceptibility $(57.10 \%)$ was observed in the second generation, whereas the sixth generation recorded minimum susceptibility $(26.66 \%)$ (Figure 1$)$. 
Table 1. Longevity assessment in subsequent generations of adult DDT-resistant colony (DRC) Phlebotomus argentipes

\begin{tabular}{lcc}
\hline & $\begin{array}{r}\text { Mean longevity of days } \pm \text { standard deviation for emerged } \\
\text { adult } P \text {. argentipes }(\text { mean } 95 \% \mathrm{CI} ; P<0.01)\end{array}$ \\
\cline { 2 - 3 } Generation(s) & Male & Female \\
\hline Control (NRC) & $7 \pm 3.5$ & $9.8 \pm 4.5$ \\
DRC-F1 & $3.8 \pm 1.0$ & $8 \pm 3.80$ \\
DRC-F2 & $4.1 \pm 1.0$ & $5 \pm 0.5$ \\
DRC-F3 & $5 \pm 0.5$ & $8 \pm 2.0$ \\
DRC-F4 & $9 \pm 4.5$ & $13.5 \pm 3.0$ \\
DRC-F5 & $3.8 \pm 2.0$ & $7 \pm 2.5$ \\
DRC-F6 & $6 \pm 0.5$ & $10 \pm 1.0$ \\
DRC-F7 & $3 \pm 2.0$ & $10 \pm 1.0$ \\
\hline
\end{tabular}

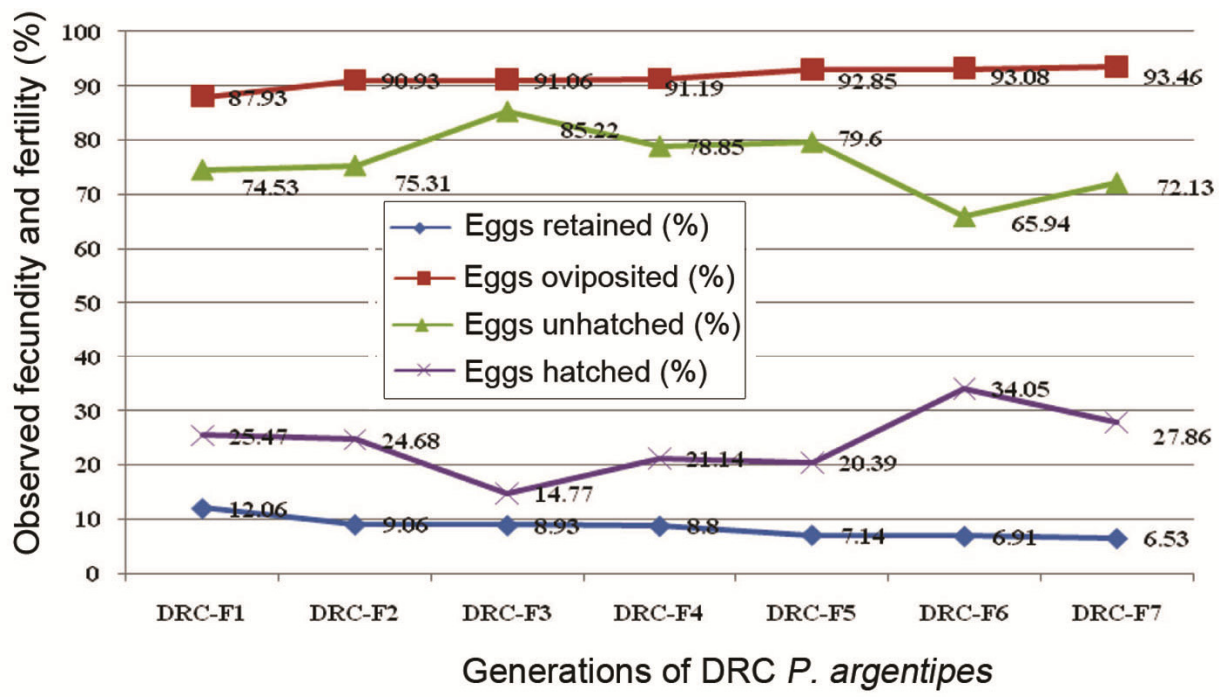

Figure 2. Comparison-based fecundity and fertility assessment in subsequent generations of DRC P. argentipes.

Under longevity analysis for adult DRC P. argentipes in the subsequent generations, mean longevity for adult female sand flies was always observed to be significantly higher (viz. $P<0.01$; CI 95\%) than that for adult male sand flies throughout the seventh generation. For female sand flies, minimum $(5 \pm 0.5)$ and maximum $(13.5 \pm 3.0)$ mean longevity was recorded during the second and fourth generation respectively, whereas minimum $(3 \pm 2.0)$ and maximum $(9 \pm 4.5)$ mean longevity was recorded for male sand flies during the seventh and fourth generation respectively (Table 1 ).

\section{Fecundity and fertility assessment}

Under the fecundity assessment of DRC sand flies in subsequent generations post DDT exposure, various fecundity factors, viz. number of egg follicles developed and successfully oviposited, those failed to oviposit by the females and retained within them in their ovary, hatchings, non-hatchings, etc. were monitored and recorded.
The number of eggs oviposited plus the eggs retained by female insects denoting total or potential fecundity, indicates the reproductive capacity of female insects (the number of eggs or mature embryos within the reproductive tract $)^{29,30}$, whereas the number of offspring actually produced by them during their lifetime corresponds to the realized or ovipositional fecundity ${ }^{31}$. Potential fecundity serves as a good indicator of future reproductive output, but in some cases the difference between potential and realized fecundity can be substantial.

In the present study, potential fecundity (eggs oviposited + eggs retained) for DDT-exposed sand flies in the subsequent generations, was observed to decrease from 2429 (in the first generation) to 1148 (in the seventh generation), in contrast to ovipositional fecundity (viz. percentage of eggs oviposited by female sand flies) that was observed to increase from $87.93 \%$ (in the first generation) to $93.46 \%$ (in the seventh generation). Whereas mean \pm percentage for egg retention per ovipositing sandfly was observed to decrease from $7.32 \% \pm 12.06 \%$ (in the first generation) to $0.42 \% \pm 6.53 \%$ (in seventh 
RESEARCH ARTICLES

Table 2. Detailed fecundity and fertility assessment in subsequent generations of female DRC P. argentipes

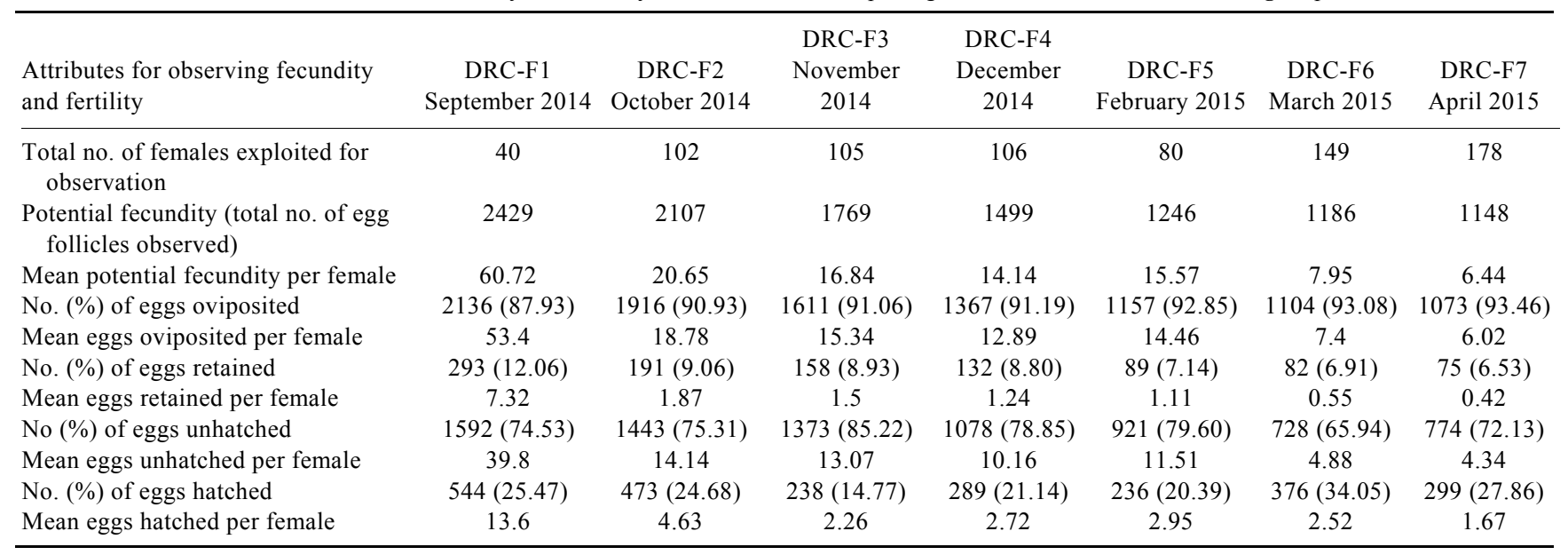

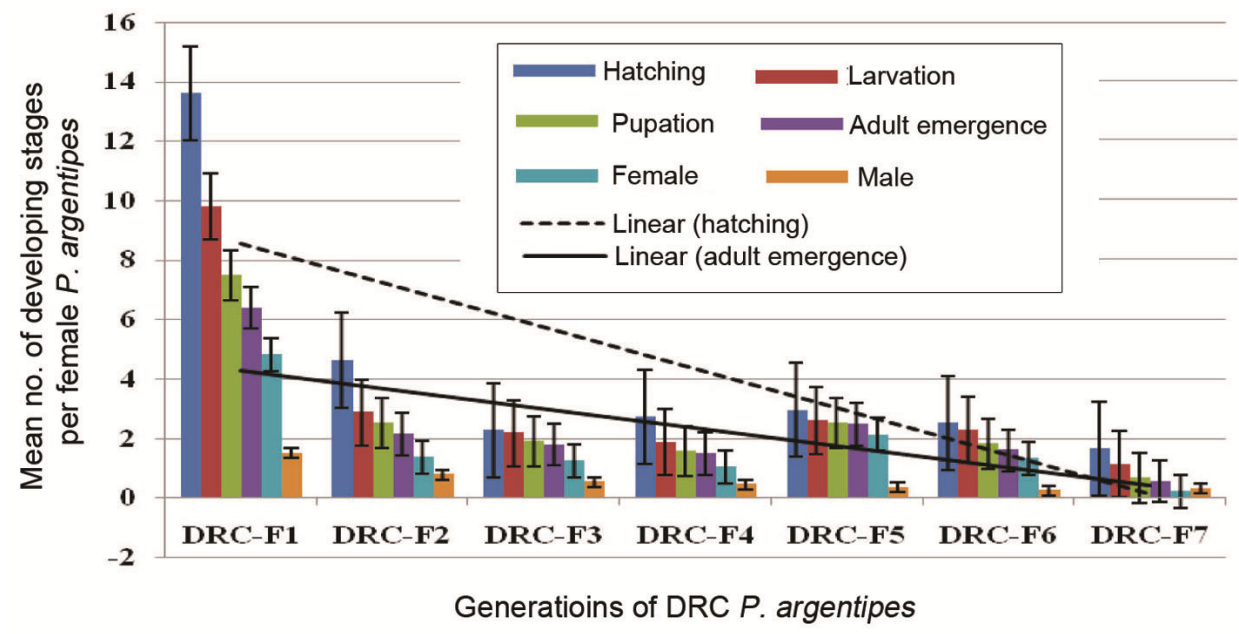

Figure 3. Comparison-based growth trends for developing stages in subsequent generations of DRC P. argentipes.

generation). However, there were fluctuations in hatching representing fertility of female sand flies due to many associated factors responsible for hatching of eggs, viz. temperature, humidity, moisture, $\mathrm{pH}$ of ovipositing surface, etc. Figure 2 and Table 2 provide the comparison based on detailed fecundity and fertility assessment in subsequent generations of female DRC P. argentipes.

\section{Fitness profile of DRC sand flies}

Figure 3 and Table 3 illustrate life-table attributes and growth trends in subsequent generations of DRC sand flies post exposure to DDT.

In the life-table assessment process, growth time (days) represented by mean \pm standard deviation for each developing stage of DRC sand flies, post insecticide exposure of adults, was scrupulously monitored till the seventh generation. For this, the developmental time taken by the female sand flies for oviposition following blood meal, and then from egg-to-adult emergence as well as the time taken by their intermediary stages, i.e. larvae and pupae, served as the developmental attributes for assessing the fitness profile of DRC sand flies.

The mean generation time (days) taken by adult female (mother) sand flies for enduring egg deposition after their successful blood feeding and finally leading to adult emergence from eggs laid by them, for their next generation, was recorded to be minimum $(29.1 \pm 0.03)$ and maximum $(51.4 \pm 5.3)$ in the second and the fifth generation respectively. Whereas the mean number of days taken by the developing stages right from the hatching of eggs to the adult phase was also recorded to be minimum $(25 \pm 3.7)$ and maximum $(44.6 \pm 3.5)$ in the second and fifth generation respectively. Also, no significant difference was observed between the mean generation time (days) for emergence of adult female (32.20 \pm 3.2$)$ and male $(32.41 \pm 2.1)$ at $\mathrm{CI}$ of $95 \%$ till the seventh generation. 


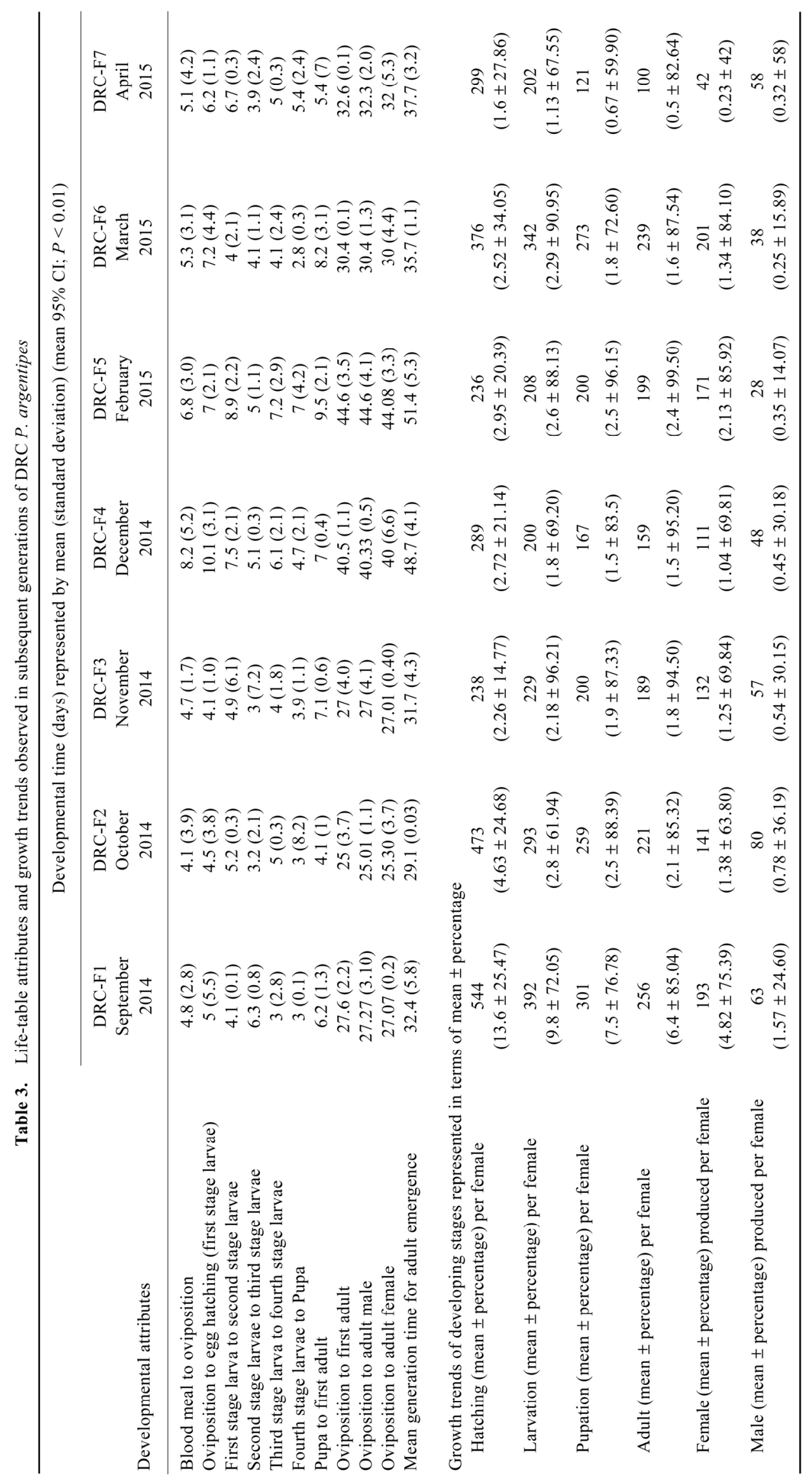


Table 4. Results for assessing blood and sucrose feeding pattern in DRC sand flies at different timescales after completion of DDT susceptibility test

\begin{tabular}{|c|c|c|c|c|}
\hline \multirow{3}{*}{$\begin{array}{l}\text { Timescale for observation } \\
\text { after completion of DDT } \\
\text { susceptibility test }\end{array}$} & \multicolumn{4}{|c|}{ Feeding pattern post-DDT exposure } \\
\hline & \multicolumn{2}{|c|}{ Blood feeding status } & \multicolumn{2}{|c|}{ Sucrose feeding status } \\
\hline & Observed fed/total & Feeding percentage & Observed fed/total & Feeding percentage \\
\hline After third hour & $15 / 150$ & 10 & $6 / 150$ & 4 \\
\hline After sixth hour & $69 / 150$ & 46 & $72 / 150$ & 48 \\
\hline After 12th hour & $117 / 150$ & 78 & $90 / 150$ & 60 \\
\hline After 24th hour & $123 / 150$ & 82 & $63 / 150 ; 87 d$ & 42 \\
\hline After 36th hour & $60 / 150 ; 90^{\mathrm{d}}$ & 40 & $42 / 150 ; 108 \mathrm{~d}$ & 28 \\
\hline
\end{tabular}

${ }^{\mathrm{d}}$ Death of test samples during the experimental process.

Table 5. Comparison-based post-feeding survivorship of adult DRC P. argentipes

\begin{tabular}{|c|c|c|c|}
\hline \multirow[b]{2}{*}{ Sand flies } & \multicolumn{3}{|c|}{$\begin{array}{l}\text { Mean }( \pm \mathrm{SE}) \text { survivorship (days) post-meal supplementation } \\
\qquad(\text { mean } 95 \% \mathrm{CI} ; P<0.01)\end{array}$} \\
\hline & Sucrose-fed male & Sucrose-fed female & Blood-fed female \\
\hline Control NRC & $10.32 \pm 5.0^{\mathrm{a}}$ & $10.01 \pm 2.0^{\mathrm{b}}$ & $18.80 \pm 4.0^{\mathrm{b}}$ \\
\hline Test DRC sand (different generations) & $10.33 \pm 0.5^{\mathrm{e}}$ & $8 \pm 3.0^{\mathrm{d}}$ & $16.24 \pm 1.0^{\mathrm{e}}$ \\
\hline
\end{tabular}

Note: Values are the mean for 300 sand flies tested in three batches of experiments. Mean in each column having different letter(s) differs significantly at $0.05 \%$ (DMRT).

Following the growth trends for the developing stages and observing the mean number of developing stages per female sandfly, the mean hatching per female was observed to decrease from the first generation $(13.6 \% \pm$ $25.47 \%)$ to the seventh generation $(1.6 \% \pm 27.86 \%)$. Likewise, larvation $(9.8 \% \pm 72.05 \%$ in the first generation and $1.13 \% \pm 67.55 \%$ in the seventh generation) and pupation $(7.5 \pm 76.78 \%$ in the first generation and $0.67 \% \pm 59.90 \%$ in the seventh generation) followed similar decreasing growth trends.

Also, mean adult emergence was always observed to be comparatively lower than the egg hatching, larvation or pupation, in a generation. The mean adult female emergence per parent female sandfly displayed fluctuating trend, but it was always comparatively higher than that of male sand flies, throughout the seventh generation (Figure 3).

\section{Feeding pattern and post-feeding survivorship of resistant sand flies}

Experiment on feeding pattern, preferences and postfeeding survivorship of DRC sand flies at different timescales following completion of susceptibility test against DDT, demonstrated maximum feeding percentage at 24th $\mathrm{h}(82 \%)$ and 12 th $\mathrm{h}(60 \%)$ for blood meal source and sucrose source respectively. However, at the 24 th h, sand flies successfully tolerated insecticide pressure and started 'falling off' due to no or very poor consumption of either of the provided energy supplements, i.e. blood or sucrose (Table 4).
Comparative evaluation of post-feeding survival of adult DRC sand flies, female $P$. argentipes benefitted with blood meal, were observed to be less survived $(16.24 \pm 1.0)$ compared with the control $(18.80 \pm 4.0)$, but it was far more than either sucrose-fed female sand flies $(8 \pm 3.0)$ or sucrose-fed male sand flies $(10.33 \pm 0.5)$. Similar to the blood meal observation, sucrose-fed female sand flies demonstrated decreasing trend $(8 \pm 3.0)$ when compared to the control $(10.01 \pm 2.0)$. Whereas insecticide exposure does not lead to any serious differences among sucrose-fed DRC male sand flies $(10.33 \pm 0.5)$ compared to the control $(10.32 \pm 5.0)$ (Table 5).

\section{Discussion}

Continuous and injudicious exploitation of DDT for checking out the incrimination manifested by $P$. argentipes in India has resulted in resistance among them in such a way, that the population has now adapted itself showing behavioural transition, including host preference and selection ${ }^{3}$, following enhanced survivorship under insecticide pressure ${ }^{13}$. The insecticide-mediated behavioural shifting, i.e. behavioural responses triggered by insecticides among the targeted population of flies, is thus likely to affect ongoing chemical-based strategies for their control as well as all aspects of VL control policies implemented at the grass-root level. Though research on fitness cost effects on DDT-resistant mosquitoes was conducted way back in 1948 (refs 26, 27, 36), it is still neglected in case of VL vector species. In this regard, the present study was designed to strengthen knowledge 
regarding the probable side effects associated with DDT resistance in the form of affected physiological fitness and reproductive robustness in subsequent generations concerning the VL vector species.

Susceptibility confirmation establishes the intrusion of susceptibility factor in DDT-resistant colony of sand flies and though incriminated with insecticide susceptibility, resistance still dominates within the colony ${ }^{12}$. Previous reports confirm DDT resistance and lethal time of exposure by the resistant sand flies to be directly proportional to each other, and thus help them to survive better ${ }^{4,8,12,32,33}$. The results of longevity analyses in the present study reveal maximum mean longevity for DRC female sand flies, i.e. $13.5 \pm 3.0$ to be comparatively higher than that of the male, i.e. $9 \pm 4.5$ till the seventh generation, corresponding to the established hypothesis of increased lethal time by the resistant female sand flies followed by increased resistance factor among them ${ }^{13}$. However, with increased resistance, the bioassay observation of least endorsement of either blood or sucrose meal by DRC sand flies, reveals sand flies enduring insecticide pressure successfully, with major changes in their normal feeding pattern, such that despite their longer period of starvation, they fail to supplement themselves with their preferred meal at the proper time, ultimately leading to death. These results corroborate with the documented hypothesis of increased resistant factors in $P$. argentipes in parallel affecting the host preferences and feeding pattern in a negative $\operatorname{sense}^{3}$, and thus decline post-feeding survivorship of adult DRC sand flies as well as growth trends, i.e. hatching, larvation, pupation ultimately affecting the adult emergence in forthcoming generation. Though we did not estimate disease transmission by DRC sand flies, the experimental observations establish reduced potential fecundity, egg retention per ovipositing sand flies and insecticide-induced fertility in the subsequent generations affecting the fitness of individuals. Forthcoming generations might have implications for insecticide exposure affecting the quality of VL vector population in terms of their fitness and fecundity as well as expected chances of VL transmission ${ }^{34}$.

Therefore, coping with insecticide toxicity assures increased life expectancy among the female insect population and thus increases the chances of disease transmission by them ${ }^{35,36}$. It also affects the quality of potent vector by affecting the physiological and behavioural temperament in terms of LF3 among the vector population. The changed physiology, feeding preferences and oviposition behaviour of the vector population are associated with the evolution of resistance, as insecticides tend to target the insect nervous system where mutations conferring resistance can have pleiotropic effects on nerve function and insect behaviour ${ }^{36,37}$. These effects on behaviour may lead to changes in the success of insects under natural conditions, thus imposing indirect fitness costs caused by IR.

\section{Conclusion}

Insecticidal exposure harnessing possible changes in the reproductive biology of $P$. argentipes has important and effective implications on vector control, as a reduced egg yield causes lower population numbers and therefore decreases the likelihood of disease transmission caused by the vector. If these effects persist in nature, impact of IR over disease management may not be as detrimental as previously considered. However, increased disease transmission following increased vectorial capacity may also be expected in the absence of DDT. Therefore, consequences of DDT resistance for disease ecology deserve additional attention.

\section{Competing interests: None to declare}

1. Swaminath, C. S., Shortt, H. E. and Anderson, L. A., Transmission of Indian kala-azar to man by the bites of Phlebotomus argentipes. Indian J. Med. Res., 1942, 30, 473-477.

2. Kishore, K., Kumar, V., Kesari, S. and Dinesh, D. S., Vector control in leishmaniasis. Indian J. Med. Res., 2006, 123(3), 467.

3. Kumar, V., Shankar, L., Rama, A., Kesari, S., Dinesh, D. S., Bhunia, G. S. and Das, P., Analysing host preference behavior of Phlebotomus argentipes (Diptera: Psychodidae) under the impact of indoor residual spray. IJTDH, 2015, 7(2), 69-79.

4. Kishore, K., Kumar, V., Kesari, S., Bhattacharya, S. K. and Das, P., Susceptibility of Phlebotomus argentipes against DDT in endemic districts of North Bihar, India. J. Commun. Dis., 2004, 36(1), 41-44.

5. Kumar, V. et al., A report on the indoor residual spraying (IRS) in the control of Phlebotomus argentipes, the vector of visceral leishmaniasis in Bihar (India): an initiative towards total elimination targeting 2015 (series-1). J. Vector Borne Dis., 2009, 46(3), 225.

6. Joshi, A. et al., Can visceral leishmaniasis be eliminated from Asia? J. Vector Borne Dis., 2008, 45(2), 105.

7. Muniaraj, M., The lost hope of elimination of kala-azar (visceral leishmaniasis) by 2010 and cyclic occurrence of its outbreak in India, blame falls on vector control practices or co-infection with human immunodeficiency virus or therapeutic modalities? Trop. Parasitol., 2014, 4(1), 10.

8. Mukhopadhyay, A. K., Saxena, N. B. and Narasimham, M. V., Susceptibility status of Phlebotomus argentipes to DDT in some kala-azar endemic areas of Bihar (India). Indian J. Med. Res., 1990, 91, 458-460.

9. Singh, R., Das, R. K. and Sharma, S. K., Resistance of sand flies to DDT in kala-azar endemic districts of Bihar, India. Bull. WHO, 2001, 79(8), 793.

10. Coleman, M. et al., DDT-based indoor residual spraying suboptimal for visceral leishmaniasis elimination in India. Proc. Natl. Acad. Sci. USA, 2015, 112(28), 8573-8578.

11. Kumar, V., Shankar, L., Kesari, S., Bhunia, G. S., Dinesh, D. S., Mandal, R. and Das, P., Insecticide susceptibility of Phlebotomus argentipes and assessment of vector control in two districts of West Bengal, India. Indian J. Med. Res., 2015, 142(2), 211.

12. Rama, A., Kumar, V., Kesari, S., Singh, V. P. and Das, P., Monitoring Susceptibility Status of Phlebotomus argentipes (Diptera: Psychodidae) at Bihar (India) for the procurement of homozygous DDT resistant colony. J. Trop. Dis., 2015, 3(4), 170.

13. Rama, A., Kesari, S., Das, P. and Kumar, V., Studying DDT susceptibility at discriminating time interval focusing maximum limit of exposure time survived by DDT resistant Phlebotomus 
argentipes (Diptera: Psychodidae) - an investigatory report. Jpn. J. Infect Dis., 2017, 70(4), 437-441.

14. Kumar, V. et al., Investigating associative impact of indoor residual spray and insecticide treated nets for minimizing visceral leishmaniasis vector population in Bihar (India). IJTDH, 2017, $\mathbf{2 3}(4), 1-15$.

15. National Vector Borne Disease Control Programme. Accelerated plan for kala-azar elimination 2017 - a guideline by National Vector Borne Disease Control Programme (NVBDCP), 2017.

16. World Health Organization. WHO Regional Committee for SouthEast Asia. Report of the Sixty-seventh Session, Dhaka, Bangladesh, 9 to 12 September 2014. World Health Organization, Regional Office for South-East Asia, New Delhi, India, 2015, pp. $1-149$.

17. Lainson, R. and Rangel, E. F., Lutzomyia longipalpis and the ecoepidemiology of American Visceral Leishmaniasis, with particular reference to Brazil: a review. Mem. Inst. Oswaldo Cruz., 2005, 100(8), 811-827.

18. Kumar, V., Kesari, S., Kumari, K., Krishnakumari, B., Venugopalan, R. and Das, P., Comparison of in vivo host animals as bloodfeeding source for laboratory rearing of the sand fly vector Phlebotomus argentipes (Diptera: Psychodidae). Annu. Entomol. Soc. Am., 2011, 104(3), 429-433.

19. Modi, G. B. and Tesh, R. B., A simple technique for mass rearing Lutzomyia longipalpisand Phlebotomus papatasi (Diptera: Psychodidae) in the laboratory. J. Med. Entomol., 1983, 20(5), 568569.

20. Rama, A., Kesari, S., Dinesh, D. S., Seema, K., Das, P. and Kumar, V., Vertebrate excreta based semiochemical influencing oviposition and neonates' survival in Phlebotomus argentipesVisceral Leishmaniasis vector in Indian subcontinent. JEZS, 2014, 2(6), 172-178.

21. World Health Organization, Instructions for determining the susceptibility or resistance of adult mosquitoes to organochlorine, organophosphate and carbamate insecticides-diagnostic test. World Health Organization, Geneva, 1981.

22. Lawyer, P. G., Rowton, E. D., Perkins, P. V., Johnson, R. N. and Young, D. G., Recent advances in laboratory mass rearing of phlebotomine sand flies. Parassitologia, 1991, 33, 361-364.

23. Modi, G. B. and Rowton, E. D., Laboratory maintenance of phlebotomine sand flies. In Maintenance of Human, Animal, and Plant Pathogen Vectors (eds Maramorosch, K. and Mahmood, F.), Oxford and IBH Publishing Co Pvt Ltd, New Delhi, Chapter 5, 1999, pp. 107-121.

24. Farias, E. D. S., Alencar, R. B., Justiniano, S. B., Freitas, R. A. D., Coelho, L. D. S., Rios-Velasquez, C. M. and Pessoa, F. A. C., The genital atrium armature of some Brazilian sand fly females (Diptera: Phlebotominae). Acta Amaz., 2015, 45(1), 81-88.

25. Kumari, S., Rama, A., Kesari, S., Purkait, B., Das, P. and Kumar, $\mathrm{V}$., Armature of genital atrium: an important tool of parity indicator and age grading in Phlebotomusargentipes (Diptera: Psychodidae) the vector of Indian kala-azar. Int. J. Mosquito Res., 2016, 3(5), 01-04.

26. Martins, A. J., Ribeiro, C., De, M., Bellinato, D. F., Peixoto, A. A., Valle, D. and Lima, J. B. P., Effect of insecticide resistance on development, longevity and reproduction of field or laboratory selected Aedes aegypti populations. PLoS ONE, 2012, 7(3), e31889; https://doi.org/10.1371/journal.pone.0031889.
27. Tabbabi, A. and Ben, BenCheikh, H., Fitness cost in laboratory selected strain of the potential mosquito vector of West Nile Virus (Culex pipiens) associated with resistance to the insecticide Temephos. J. Middle East North Africa Sci., 2017, 3(5), 10-15.

28. Kumar, V., Krishnakumari, B., Kesari, S., Kumari, K., Kumar, R., Ranjan, A. and Das, P., Preliminary observations on the female behavior of the Indian sand fly vector. Phlebotomus argentipes (Diptera: Psychodidae). Ann. Entomol. Soc. Am., 2012, 105(2), 201-205.

29. Bintcliffe, E. J. B. and Wratten, S. D., Antibiotic resistance in potato cultivars to the aphid Myzuspersicae. Ann. Appl. Biol., 1982, 100, 383-391.

30. Kazimirova, M., Influence of larval crowding and mating on lifespan and fecundity of Mamestra brassicae (Lepidoptera: Noctuidae). Eur. J. Entomol., 1996, 93(1), 45-52.

31. Leather, S. R., Factors affecting fecundity, fertility, oviposition, and larviposition in insects. In Insect Reproduction (eds Leather, S. R. and Hardie, J.), CRC Press, Boca Raton, 1995, pp. 143-174.

32. Dhiman, R. C., Raghavendra, K., Kumar, V., Kesari, S. and Kishore, K., Susceptibility status of Phlebotomus argentipes to insecticides in districts Vaishali and Patna (Bihar). J. Commun. Dis., 2003, 35(1), 49-51.

33. Singh, R. and Kumar, P., Susceptibility of the sand fly Phlebotomus argentipes Annandale and Brunetti (Diptera: Psychodidae) toInsecticides in Endemic Areas of Visceral leishmaniasis in Bihar, India. Jpn. J. Infect. Dis., 2015, 68, 33-37.

34. Lane, R. P., The contribution of sand fly control to leishmaniasis control. Ann. Soc. Belg. Med. Trop., 1991, 71(1), 65-74.

35. Busvine, J. R., The significance of insecticide-resistant strains, with special reference to pests of medical importance. Bull. Org. mond. Sante., 1956, 15(3-5), 389-401. Bull. Wld. Hlth. Org. 1.

36. Desneux, N., Decourtye, A. and Delpuech, J. M., The sublethal effects of pesticides on beneficial arthropods. Annu. Rev. Entomol., 2007, 52, 81-106.

37. Lee, C. Y., Sublethal effects of insecticides on longevity, fecundity and behaviour of insect pests: a review. J. Biosci., 2000, 11(1), $107-112$.

ACKNOWLEDGEMENTS. We thank N. K. Sinha, S. A. Khan, late A. K. Mandal, B. B. Prasad and staff of the Department of Vector Biology and Control, ICMR-RMRIMS for technical support. We also thank SAC, Institutional Ethics Committee and the Animal Ethics Committee (AEC) of ICMR-RMRIMS for their mandatory approval to conduct this study. This work is part of a Ph D programme at the University of Calcutta, Kolkata, financially sponsored by University Grant Commission, New Delhi (grant number RGNF 2012-13-ST-BIH20305), and a study at ICMR-RMRIMS, Patna (Id. no. INT-119VBC/2015) duly approved by the Scientific Advisory Committee (SAC) of ICMR-RMRIMS in 2015.

Received 12 February 2018; revised accepted 30 April 2020

doi: $10.18520 / \mathrm{cs} / \mathrm{v} 119 / \mathrm{i} 1 / 103-111$ 